JOURNAL OF THE

AMERICAN MATHEMATICAL SOCIETY

Volume 22, Number 2, April 2009, Pages 331-352

S 0894-0347(08)00616-4

Article electronically published on September 24, 2008

\title{
INVARIANCE OF TAUTOLOGICAL EQUATIONS II: GROMOV-WITTEN THEORY
}

\author{
Y.-P. LEE, WITH APPENDIX A BY Y. IWAO AND Y.-P. LEE
}

\section{INTRODUCTION}

0.1. Gromov-Witten theory and moduli of curves. This work is a continuation of Part I of ITE [16. The purpose of this paper is to explore the technique of invariance of tautological equations in the realm of Gromov-Witten theory.

In Part I, the main focus was to study the linear invariance operators

$$
\mathfrak{r}_{l}: R^{k}\left(\bar{M}_{g, n}^{\bullet}\right) \rightarrow R^{k+l-1}\left(\bar{M}_{g-1, n+2}^{\bullet}\right)
$$

between the tautological rings of moduli of curves. Here • stands for possibly disconnected curves. Note that the arithmetic genus for disconnected curve is defined to be

$$
g(C):=\sum_{i=1}^{d} g\left(C_{i}\right)-d+1,
$$

where $C_{i}$ are connected components of $C, C=\amalg_{i=1}^{d} C_{i}$. By the definition given in [16], the curves in the image of $\mathfrak{r}_{l}$ have at most one more connected component. Therefore, the connected components of the image curves would have either smaller genus or the same genus but less marked points than those of the domain curves. It is therefore easy to see that these operators give an algorithm in producing tautological relations inductively. This was explained in Part I.

In Part II, the focus moves to Gromov-Witten theory. One of the main themes of the current work is to study the interplay between Gromov-Witten theory and the moduli of curves. The idea of studying and utilizing this interaction is not new. One such example is given by the localization technique in Gromov-Witten theory. The fixed point loci on moduli of stable maps to projective spaces are moduli of curves (or more precisely their quotients by finite groups). Therefore, the known results on moduli of curves can be used to calculate Gromov-Witten invariants. Conversely, by intelligently playing with the "trivial" identities on moduli of maps, one can produce "non-trivial" identities on their fixed point loci. See 21 for a nice survey on this subject. Here however Gromov-Witten theory is used in a different way. Roughly, instead of localization, deformation theory of Gromov-Witten theory is used. Along the way, some results in Gromov-Witten theory are also proved.

In order to apply deformation theory of Gromov-Witten theory, Givental's axiomatic framework is introduced. Givental discovered a twisted loop group action

Received by the editors May 30, 2006.

2000 Mathematics Subject Classification. Primary 14N35, $14 \mathrm{H} 10$.

Key words and phrases. Gromov-Witten theory, moduli of curves.

This research was partially supported by NSF and an AMS Centennial Fellowship.

(C)2008 American Mathematical Society Reverts to public domain 28 years from publication 
on the moduli space of Frobenius manifolds (or genus zero Gromov-Witten theories) and proved that the semisimple theories (of a given fixed dimension) form a single orbit of the group action; see Theorem 1. Furthermore, quantization of the group action on the semisimple orbit is shown to carry the corresponding higher genus Gromov-Witten theory from one to another [11, 12, 20; see Theorem 2. The operators $\mathfrak{r}_{l}$ form the Lie algebra of this quantized group action.

Due to the existence of the stablization morphisms (3) from moduli of maps to moduli of curves, tautological relations on moduli of curves can be pulled-back to any geometric Gromov-Witten theory. It is reasonable to expect that the tautological relations also holds in axiomatic Gromov-Witten theories, and the twisted loop group action preserve the relations. That is, the equations of the tautological classes induced from moduli of curves should be invariant under the the twisted loop group action. This is the origin of the invariance of tautological equations.

0.2. Outline of the contents. Section 1 gives a quick summary of geometric Gromov-Witten theory, mainly to fix the notation. Givental's axiomatic GromovWitten theory, in genus zero and higher genera, is summarized in Sections 2 and 3. Some geometric results on the tautological classes, surely known to experts, are given in Section 4 for the readers' convenience. In Section 5, it is proved that each tautological class is invariant under the quantized lower triangular loop groups. In Section 6, we study the invariance of tautological equations under the quantized upper triangular loop groups. At the end of the paper, we indicate a few applications, including a Faber type statement for the tautological rings [3] and a uniform derivation of all known tautological equations [13, 1, 2]. An appendix (jointly with Y. Iwao) demonstrates some properties of the upper triangular loop groups associated to $\mathbb{P}^{1}$.

\subsection{Later developments.}

0.3.1. Geometric interpretation and work of Faber-Shadrin-Zvonkine. The operators $\mathfrak{r}_{l}$ are defined in Part I via operations on the decorated graphs. Note that one class in $R^{k}\left(\bar{M}_{g, n}\right)$ may have more than one graphical presentation due to the existence of tautological relations. The fact that certain combinations of these graphical operations descend to operations on $R^{k}\left(\bar{M}_{g, n}\right)$ is shown originally through GromovWitten theory, via complicated reduction. It was later realized, independently by Pandharipande with myself, and by Faber-Shadrin-Zvonkine [6], that they are consequences of simple geometric operations on moduli of curves. (Roughly, these operations are pull-backs of tautological classes via gluing morphisms.) The geometric interpretation easily confirms Conjecture 1 in Part I, while falsifying the other 2 conjectures. See Section 3 of $[6$ for details. As an application, FaberShadrin-Zvonkine showed that Witten's conjecture on $r$-spin curves follows from their earlier works. Indeed, 6] contains a well-written and slightly different perspective of the current work and is recommended to the readers of this paper.

0.3.2. Gromov-Witten classes instead of invariants. In light of C. Teleman's work [20], which confirms Givental's conjecture at the level of cohomology classes, the current work can and should be rephrased in terms of Gromov-Witten classes, defined [14, instead of invariants. It is not difficult to see that results here hold in the more general framework. 


\section{Geometric Gromov-Witten theory}

1.1. Preliminaries of Gromov-Witten theory. Gromov-Witten theory studies the tautological intersection theory on $\bar{M}_{g, n}(X, \beta)$, the moduli spaces of stable maps from curves $C$ of genus $g$ with $n$ marked points to a smooth projective variety $X$. The intersection numbers, or Gromov-Witten invariants, are integrals of tautological classes over the virtual fundamental classes of $\bar{M}_{g, n}(X, \beta)$ :

$$
\int_{\left[\bar{M}_{g, n}(X, \beta)\right] \operatorname{vir}} \prod_{i=1}^{n} \operatorname{ev}_{i}^{*}\left(\gamma_{i}\right) \psi_{i}^{k_{i}} .
$$

Here $\gamma_{i} \in H^{*}(X)$ and $\psi_{i}$ are the cotangent classes (gravitational descendents).

For the sake of the later reference, let us fix the notation.

(i) $H:=H^{*}(X, \mathbb{Q})$ is a $\mathbb{Q}$-vector space, assumed of $\operatorname{rank} N$. Let $\left\{\phi_{\mu}\right\}_{\mu=1}^{N}$ be a basis of $H$.

(ii) $H$ carries a symmetric bilinear form, Poincaré pairing,

$$
\langle\cdot, \cdot\rangle: H \otimes H \rightarrow \mathbb{Q} .
$$

Define

$$
g_{\mu \nu}:=\left\langle\phi_{\mu}, \phi_{\nu}\right\rangle
$$

and $g^{\mu \nu}$ to be the inverse matrix.

(iii) Let $\mathcal{H}_{t}:=\bigoplus_{k=0}^{\infty} H$ be the infinite dimensional complex vector space with basis $\left\{\phi_{\mu} \psi^{k}\right\}$. $\mathcal{H}_{t}$ has a natural $\mathbb{Q}$-algebra structure,

$$
\phi_{\mu} \psi^{k_{1}} \otimes \phi_{\nu} \psi^{k_{2}} \mapsto\left(\phi_{\mu} \cdot \phi_{\nu}\right) \psi^{k_{1}+k_{2}},
$$

where $\phi_{\mu} \cdot \phi_{n u}$ is the cup product in $H$.

(iv) Let $\left\{t_{k}^{\mu}\right\}, \mu=1, \ldots, N, k=0, \ldots, \infty$, be the dual coordinates of the basis $\left\{\phi_{\mu} \psi^{k}\right\}$.

We note that at each marked point, the insertion is $\mathcal{H}_{t}$-valued. Let

$$
t:=\sum_{k, \mu} t_{k}^{\mu} \phi_{\mu} \psi^{k}
$$

denote a general element in the vector space $\mathcal{H}_{t}$. To simplify the notation, $t_{k}$ will stand for the vector $\left(t_{k}^{1}, \ldots, t_{k}^{N}\right)$ and $t^{\mu}$ for $\left(t_{0}^{\mu}, t_{1}^{\mu}, \ldots\right)$.

(v) Define

$$
\left\langle\partial_{k_{1}}^{\mu_{1}} \ldots \partial_{k_{n}}^{\mu_{n}}\right\rangle_{g, n, \beta}:=\int_{\left[\bar{M}_{g, n}(X, \beta)\right]^{\mathrm{vir}}} \prod_{i=1}^{n} \mathrm{ev}_{i}^{*}\left(\phi_{\mu_{i}}\right) \psi_{i}^{k_{i}}
$$

and define

$$
\left\langle t^{n}\right\rangle_{g, n, \beta}=\langle t \ldots t\rangle_{g, n, \beta}
$$

by multi-linearity.

(vi) Let

$$
F_{g}^{X}(t):=\sum_{n, \beta} \frac{1}{n !}\left\langle t^{n}\right\rangle_{g, n, \beta}
$$


be the generating function of all genus $g$ Gromov-Witten invariants 11 The $\tau$-function of $X$ is the formal expression

$$
\tau_{G W}^{X}:=e^{\sum_{g=0}^{\infty} \hbar^{g-1} F_{g}^{X}} .
$$

1.2. Gravitational ancestors and the $(3 g-2)$-jet properties. Let

$$
\text { st : } \bar{M}_{g, m+l}(X, \beta) \rightarrow \bar{M}_{g, m+l}
$$

be the stabilization morphism defined by forgetting the map and

$$
\mathrm{ft}: \bar{M}_{g, m+l} \rightarrow \bar{M}_{g, m}
$$

be the forgetful morphism defined by forgetting the last $l$ points. The gravitational ancestors are defined to be

$$
\bar{\psi}_{i}:=(\mathrm{ft} \circ \mathrm{st})^{*} \psi_{i}
$$

and genus $g$ ancestor potential is defined by

$$
\bar{F}_{g}^{X}(t, s):=\sum_{m, l, \beta} \frac{Q^{\beta}}{m ! l !} \int_{\left[\bar{M}_{g, m+l}(X, \beta)\right]^{\mathrm{vir}}} \prod_{i=1}^{m} \sum_{k} t_{k}^{\mu}\left(\bar{\psi}_{i}\right)^{k} \mathrm{ev}_{i}^{*}\left(\phi_{\mu}\right) \prod_{i=m+1}^{m+l} \sum_{\mu} s^{\mu} \mathrm{ev}_{i}^{*}\left(\phi_{\mu}\right) \text {. }
$$

The following property is called the $(3 g-2)$-jet property [10]:

$$
\left.\frac{\partial^{m}}{\partial t_{k_{1}+1}^{\mu_{1}} \ldots \partial t_{k_{m}+1}^{\mu_{m}}} \bar{F}_{g}^{X}(t, s)\right|_{t_{0}=0}=0 \quad \text { for } \sum k_{i} \geq 3 g-2 .
$$

This follows from the dimension counting

$$
\operatorname{dim} \bar{M}_{g, n}=3 g-3+n .
$$

The ancestors and descendents are different, but easy to compare. Let $D_{j}$ be the (virtual) divisor on $\bar{M}_{g, n+m}(X, \beta)$ defined by the image of the gluing morphism

$$
\sum_{\beta^{\prime}+\beta^{\prime \prime}=\beta} \sum_{m^{\prime}+m^{\prime \prime}=m} \bar{M}_{0,2+m^{\prime}}^{(j)}\left(X, \beta^{\prime}\right) \times_{X} \bar{M}_{g, n+m^{\prime \prime}}\left(X, \beta^{\prime \prime}\right) \rightarrow \bar{M}_{g, n+m}(X, \beta),
$$

where $\bar{M}_{g, n+m^{\prime \prime}}\left(X, \beta^{\prime \prime}\right)$ carries all first $n$ marked points except the $j$-th one, which is carried by $\bar{M}_{0,2+m^{\prime}}^{(j)}\left(X, \beta^{\prime}\right)$. It is proved in [15] that

$$
\psi_{j}-\bar{\psi}_{j}=\left[D_{j}\right]^{\mathrm{vir}} \text {. }
$$

\section{Genus zero axiomatic Gromov-Witten theory}

Let $H$ be a $\mathbb{Q}$-vector space of dimension $N$ with a distinguished element 1 . Let $\left\{\phi_{\mu}\right\}$ be a basis of $H$ and let $\phi_{\mathbf{1}}=\mathbf{1}$. Assume that $H$ is endowed with a nondegenerate symmetric $\mathbb{Q}$-bilinear form, or metric, $\langle\cdot, \cdot\rangle$. Let $\mathcal{H}$ denote the infinite dimensional vector space $H\left[z, z^{-1}\right]$ consisting of Laurent polynomials with coefficients in $H 2$ Introduce a symplectic form $\Omega$ on $\mathcal{H}$ :

$$
\Omega(f(z), g(z)):=\operatorname{Res}_{z=0}\langle f(-z), g(z)\rangle,
$$

where the symbol $\operatorname{Res}_{z=0}$ means to take the residue at $z=0$.

There is a natural polarization $\mathcal{H}=\mathcal{H}_{q} \oplus \mathcal{H}_{p}$ by the Lagrangian subspaces $\mathcal{H}_{q}:=H[z]$ and $\mathcal{H}_{p}:=z^{-1} H\left[z^{-1}\right]$ which provides a symplectic identification of

\footnotetext{
${ }^{1}$ In Gromov-Witten theory, one usually has to deal with the coefficients in the Novikov ring. We shall not touch upon these subtleties here but refer the readers to [19].

${ }^{2}$ Different completions of $\mathcal{H}$ are used in different places. This will not be discussed in the present article. See 19 for the details.
} 
$(\mathcal{H}, \Omega)$ with the cotangent bundle $T^{*} \mathcal{H}_{q}$ with the natural symplectic structure. Also, $\mathcal{H}_{q}$ has a basis

$$
\left\{\phi_{\mu} z^{k}\right\}, \quad 1 \leq \mu \leq N, \quad 0 \leq k,
$$

with dual coordinates $\left\{q_{\mu}^{k}\right\}$. The corresponding basis for $\mathcal{H}_{p}$ is

$$
\left\{\phi_{\mu} z^{-k-1}\right\}, \quad 1 \leq \mu \leq N, \quad 0 \leq k,
$$

with dual coordinates $\left\{p_{\mu}^{k}\right\}$.

For example, let $\left\{\phi_{i}\right\}$ be an orthonormal basis of $H$. An $H$-valued Laurent formal series can be written in this basis as

$$
\begin{aligned}
\ldots+\left(p_{1}^{1}, \ldots, p_{1}^{N}\right) \frac{1}{(-z)^{2}}+\left(p_{0}^{1}, \ldots, p_{0}^{N}\right) & \frac{1}{(-z)} \\
& +\left(q_{0}^{1}, \ldots, q_{0}^{N}\right)+\left(q_{1}^{1}, \ldots, q_{1}^{N}\right) z+\ldots .
\end{aligned}
$$

In fact, $\left\{p_{k}^{i}, q_{k}^{i}\right\}$ for $k=0,1,2, \ldots$ and $i=1, \ldots, N$ are the Darboux coordinates compatible with this polarization in the sense that

$$
\Omega=\sum_{i, k} d p_{k}^{i} \wedge d q_{k}^{i}
$$

The parallel between $\mathcal{H}_{q}$ and $\mathcal{H}_{t}$ is evident, and is in fact given by the following affine coordinate transformation, called the dilaton shift:

$$
t_{k}^{\mu}=q_{k}^{\mu}+\delta^{\mu 1} \delta_{k 1} .
$$

Definition 1. Let $G_{0}(t)$ be a (formal) function on $\mathcal{H}_{t}$. The pair $T:=\left(\mathcal{H}, G_{0}\right)$ is called a $g=0$ axiomatic theory if $G_{0}$ satisfies three sets of genus zero tautological equations: the Dilaton Equation (7), the String Equation (8) and the Topological Recursion Relations (TRR) (9). We have

$$
\begin{aligned}
& \frac{\partial G_{0}(t)}{\partial t_{1}^{1}}(t)=\sum_{k=0}^{\infty} \sum_{\mu} t_{k}^{\mu} \frac{\partial G_{0}(t)}{\partial t_{k}^{\mu}}-2 G_{0}(t), \\
& \frac{\partial G_{0}(t)}{\partial t_{0}^{1}}=\frac{1}{2}\left\langle t_{0}, t_{0}\right\rangle+\sum_{k=0}^{\infty} \sum_{\nu} t_{k+1}^{\nu} \frac{\partial G_{0}(t)}{\partial t_{k}^{\nu}}, \\
& \frac{\partial^{3} G_{0}(t)}{\partial t_{k+1}^{\alpha} \partial t_{l}^{\beta} \partial t_{m}^{\gamma}}=\sum_{\mu \nu} \frac{\partial^{2} G_{0}(t)}{\partial t_{k}^{\alpha} \partial t_{0}^{\mu}} g^{\mu \nu} \frac{\partial^{3} G_{0}(t)}{\partial t_{0}^{\nu} \partial t_{l}^{\beta} \partial t_{m}^{\gamma}}, \quad \forall \alpha, \beta, \gamma, k, l, m .
\end{aligned}
$$

In the case of geometric theory, $G_{0}=F_{0}^{X}$. It is well known that $F_{0}^{X}$ satisfies the above three sets of equations (7) (8) (9). The main advantage of viewing the genus zero theory through this formulation, it seems to us, is to replace $\mathcal{H}_{t}$ by $\mathcal{H}$ where a symplectic structure is available. Therefore many properties can be reformulated in terms of the symplectic structure $\Omega$ and hence independent of the choice of the polarization. This suggests that the space of genus zero axiomatic GromovWitten theories, i.e. the space of functions $G_{0}$ satisfying the string equation, dilaton equation and TRRs, has a huge symmetry group.

Definition 2. Let $L^{(2)} G L(H)$ denote the twisted loop group which consists of $\operatorname{End}(H)$-valued formal Laurent series $M(z)$ in the indeterminate $z^{-1}$ satisfying $M^{*}(-z) M(z)=\mathbf{I}$. Here ${ }^{*}$ denotes the adjoint with respect to $(\cdot, \cdot)$.

The condition $M^{*}(-z) M(z)=\mathbf{I}$ means that $M(z)$ is a symplectic transformation on $\mathcal{H}$. 
Theorem 1 (12]). The twisted loop group acts on the space of axiomatic genus zero theories. Furthermore, the action is transitive on the semisimple theories of a fixed rank $N$.

Remarks. (i) In the geometric theory, $F_{0}^{X}(t)$ is usually a formal function in $t$. Therefore, the corresponding function in $q$ would be formal at $q=\mathbf{- 1} z$. Furthermore, the Novikov rings are usually needed to ensure the well-definedness of $F_{0}^{X}(t)$ (cf. Footnote 1).

(ii) It can be shown that the axiomatic genus zero theory over complex numbers is equivalent to the definition of abstract (formal) Frobenius manifolds, not necessarily conformal. The coordinates on the corresponding Frobenius manifold is given by the following map [5]:

$$
s^{\mu}:=\frac{\partial}{\partial t_{0}^{\mu}} \frac{\partial}{\partial t_{0}^{1}} G_{0}(t) .
$$

From now on, the term "genus zero axiomatic theory" is identified with "Frobenius manifold".

(iii) The above formulation (or the Frobenius manifold formulation) does not include the divisor axiom, which is true for any geometric theory.

(iv) Coates and Givental [4] (see also [12]) give a beautiful geometric reformation of the genus zero axiomatic theory in terms of Lagrangian cones in $\mathcal{H}$. When viewed in the Lagrangian cone formulation, Theorem 1 becomes transparent and a proof is almost immediate.

\section{QuAntization AND higher GenUs AXIOMATIC THEORY}

3.1. Preliminaries on quantization. To quantize an infinitesimal symplectic transformation, or its corresponding quadratic hamiltonians, we recall the standard Weyl quantization. A polarization $\mathcal{H}=T^{*} \mathcal{H}_{q}$ on the symplectic vector space $\mathcal{H}$ (the phase space) defines a configuration space $\mathcal{H}_{q}$. The quantum "Fock space" will be a certain class of functions $f(\hbar, q)$ on $\mathcal{H}_{q}$ (containing at least polynomial functions), with additional formal variable $\hbar$ ("Planck's constant"). The classical observables are certain functions of $p, q$. The quantization process is to find for the classical mechanical system on $\mathcal{H}$ a "quantum mechanical" system on the Fock space such that the classical observables, like the hamiltonians $h(q, p)$ on $\mathcal{H}$, are quantized to become operators $\widehat{h}\left(q, \frac{\partial}{\partial q}\right)$ on the Fock space.

Let $A(z)$ be an $\operatorname{End}(H)$-valued Laurent formal series in $z$ satisfying

$$
(A(-z) f(-z), g(z))+(f(-z), A(z) g(z))=0 ;
$$

then $A(z)$ defines an infinitesimal symplectic transformation

$$
\Omega(A f, g)+\Omega(f, A g)=0 .
$$

An infinitesimal symplectic transformation $A$ of $\mathcal{H}$ corresponds to a quadratic polynomial $P(A)$ in $p, q$ :

$$
P(A)(f):=\frac{1}{2} \Omega(A f, f) .
$$


Choose a Darboux coordinate system $\left\{q_{k}^{i}, p_{k}^{i}\right\}$. The quantization $P \mapsto \widehat{P}$ assigns

$$
\begin{aligned}
& \widehat{1}=1, \widehat{p_{k}^{i}}=\sqrt{\hbar} \frac{\partial}{\partial q_{k}^{i}}, \widehat{q_{k}^{i}}=q_{k}^{i} / \sqrt{\hbar}, \\
& \widehat{p_{k}^{i} p_{l}^{j}}=\widehat{p_{k}^{i}} \widehat{p_{l}^{j}}=\hbar \frac{\partial}{\partial q_{k}^{i}} \frac{\partial}{\partial q_{l}^{j}}, \\
& \widehat{p_{k}^{i} q_{l}^{j}}=q_{l}^{j} \frac{\partial}{\partial q_{k}^{i}}, \\
& \widehat{q_{k}^{i} q_{l}^{j}}=q_{k}^{i} q_{l}^{j} / \hbar .
\end{aligned}
$$

In summary, the quantization is the process

$$
\text { A } \quad \mapsto \quad P(A) \quad \mapsto \quad \widehat{P(A)}
$$

inf. sympl. transf. $\mapsto$ quadr. hamilt. $\mapsto$ operator on Fock sp..

It can be readily checked that the first map is a Lie algebra isomorphism: the Lie bracket on the left is defined by $\left[A_{1}, A_{2}\right]=A_{1} A_{2}-A_{2} A_{1}$ and the Lie bracket in the middle is defined by Poisson bracket

$$
\left\{P_{1}(p, q), P_{2}(p, q)\right\}=\sum_{k, i} \frac{\partial P_{1}}{\partial p_{k}^{i}} \frac{\partial P_{2}}{\partial q_{k}^{i}}-\frac{\partial P_{2}}{\partial p_{k}^{i}} \frac{\partial P_{1}}{\partial q_{k}^{i}} .
$$

The second map is not a Lie algebra homomorphism, but is very close to being one.

\section{Lemma 1.}

$$
\left[\widehat{P_{1}}, \widehat{P_{2}}\right]=\left\{\widehat{P_{1}, P_{2}}\right\}+\mathcal{C}\left(P_{1}, P_{2}\right)
$$

where the cocycle $\mathcal{C}$, in orthonormal coordinates, vanishes except

$$
\mathcal{C}\left(p_{k}^{i} p_{l}^{j}, q_{k}^{i} q_{l}^{j}\right)=-\mathcal{C}\left(q_{k}^{i} q_{l}^{j}, p_{k}^{i} p_{l}^{j}\right)=1+\delta^{i j} \delta_{k l} .
$$

Example. Let $\operatorname{dim} H=1$ and $A(z)$ be multiplication by $z^{-1}$. It is easy to see that $A(z)$ is infinitesimally symplectic.

$$
\begin{aligned}
& P\left(z^{-1}\right)=-\frac{q_{0}^{2}}{2}-\sum_{m=0}^{\infty} q_{m+1} p_{m}, \\
& \widehat{P\left(z^{-1}\right)}=-\frac{q_{0}^{2}}{2}-\sum_{m=0}^{\infty} q_{m+1} \frac{\partial}{\partial q_{m}} .
\end{aligned}
$$

Note that one often has to quantize the symplectic instead of the infinitesimal symplectic transformations. Following the common practice in physics, define

$$
\widehat{e^{A(z)}}:=e^{\widehat{A(z)}},
$$

for $e^{A(z)}$ an element in the twisted loop group.

3.2. $\tau$-function for the axiomatic theory. Let $X$ be the space of $N$ points and let $H^{N p t}:=H^{*}(X)$. Let $\phi_{i}$ be the delta-function at the $i$-th point. Then $\left\{\phi_{i}\right\}_{i=1}^{N}$ form an orthonormal basis and are the idempotents of the quantum product

$$
\phi_{i} * \phi_{j}=\delta_{i j} \phi_{i} .
$$

The genus zero potential for $N$ points is nothing but a sum of genus zero potentials of a point

$$
F_{0}^{N p t}\left(t^{1}, \ldots, t^{N}\right)=F_{0}^{p t}\left(t^{1}\right)+\ldots+F_{0}^{p t}\left(t^{N}\right) .
$$

In particular, the genus zero theory of $N$ points is semisimple. 
By Theorem 11 any semisimple genus zero axiomatic theory $T$ of $\operatorname{rank} N$ can be obtained from $H^{N p t}$ by action of an element $O^{T}$ in the twisted loop group. By Birkhoff factorization, $O^{T}=S^{T}\left(z^{-1}\right) R^{T}(z)$, where $S\left(z^{-1}\right)$ (resp. $R(z)$ ) is an matrix-valued function in $z^{-1}$ (resp. $z$ ).

In order to define the axiomatic higher genus potentials $G_{g}^{T}$ for the semisimple theory $T$, one first introduces the " $\tau$-function of $T$ ".

Definition 3 ([1] $)$. Define the axiomatic $\tau$-function as

$$
\tau_{G}^{T}:=\widehat{S^{T}}\left(\widehat{R^{T}} \tau_{G W}^{N p t}\right),
$$

where $\tau_{G W}^{N p t}$ is defined in (2). Define the axiomatic genus $g$ potential $G_{g}^{T}$ via the formula (cf. (2))

$$
\tau_{G}^{T}=: e^{\sum_{g=0}^{\infty} \hbar^{g-1} G_{g}^{T}} .
$$

Remark. (i) It is not obvious that the above definitions make sense. The function $\widehat{S^{T}}\left(\widehat{R^{T}} \tau^{N p t}\right)$ is well-defined, due to the $(3 g-2)$-jet properties (5), proved in [10] for geometric Gromov-Witten theory and in [11] in the axiomatic framework. The fact $\log \tau_{G}^{T}$ can be written as $\sum_{g=0}^{\infty} \hbar^{g-1}$ (formal function in $t$ ) is also non-trivial. The interested readers are referred to the original article [11] or [19] for details.

An immediate question regarding Definition 3. When the axiomatic semisimple theory actually comes from a projective variety $X$, is the $\tau$-function defined in the axiomatic theory the same as the $\tau$-function defined in the geometric GromovWitten theory? This is known as Givental's conjecture. Givental himself establishes the special cases when $X$ is toric Fano. Recently, C. Teleman 20 has posted a complete proof based on his classification of semisimple $2 D$ cohomological field theories 3

Theorem 2 ([11], 20]). Let $X$ be a projective variety whose quantum cohomology is semisimple; then

$$
\tau_{G}^{X}=\tau_{G W}^{X}
$$

\section{Tautological equations in Gromov-Witten theory}

As stated in the Introduction, the material here is known to experts, and is included for lack of a good general reference (known to us). Therefore, the discussions will be brief.

Due to the stabilization morphism (3), any tautological equation in $\bar{M}_{g, n}$ can be pulled back and become an equation on tautological classes on $\bar{M}_{g, n}(X, \beta)$. The $\psi$-classes can be either transformed to ancestor classes $\bar{\psi}$ or to $\psi$-classes on the moduli spaces of stable maps. Due to the functorial properties of the virtual fundamental classes, the pull-backs of the tautological equations hold for GromovWitten theory of any target space. The term tautological equations will also be used for the corresponding equations in Gromov-Witten theory and in the theory of spin curves. Equations in Gromov-Witten theory which are valid for all target spaces are called the universal equations. Tautological equations are universal equations.

However, these induced equations will produce relations among generalized Gromov-Witten invariants, which involve not only $\psi$-classes but also $\kappa$-classes and

\footnotetext{
${ }^{3}$ Teleman also informed us that M. Kontsevich has a related result which gives a full description of the deformation theory of (semisimple) $2 D$ cohomological field theory.
} 
boundary classes. Since the integration over boundary classes can be written in terms of ordinary Gromov-Witten invariants by the splitting axiom, what is really in question is the $\kappa$-classes. We will start with some results in tautological classes on moduli of curves.

Let

$$
\mathrm{ft}_{l}: \bar{M}_{g, n+l} \rightarrow \bar{M}_{g, n}
$$

be the forgetful morphism, forgetting the last $l$ marked points.

\section{Lemma 2.}

$$
\left(\mathrm{ft}_{l}\right)_{*}\left(\prod_{i=1}^{n}\left(\psi_{i}\right)^{k_{i}} \prod_{i+n+1}^{n+l}\left(\psi_{i}\right)^{k_{i}+1}\right)=K_{k_{n+1} \ldots k_{n+l}} \prod_{i=1}^{n} \psi_{i}^{k_{i}},
$$

where $K_{k_{n+1} \ldots k_{n+l}}$ is defined as follows. Let $\sigma \in S_{l}$ be an element in the symmetric group permuting the set $\{n+1, \ldots n+l\}$. Then $\sigma$ can be written as a product of disjoint cycles

$$
\sigma=c^{1} c^{2} \ldots, \text { where } c^{i}:=\left(c_{1}^{i} \ldots c_{a^{i}}^{i}\right)
$$

with $c_{j}^{i} \in\{n+1, \ldots, n+l\}$. Define

$$
K_{k_{n+1} \ldots k_{n+l}}:=\sum_{\sigma \in S_{l}} \prod_{i\left(\sigma=\prod c^{i}\right)} \sum_{j=1}^{a^{i}} \kappa_{c_{j}^{i}} .
$$

For example, when $l=2$, the formula becomes

$$
\begin{aligned}
& \left(\mathrm{ft}_{2}\right)_{*}\left(\left(\prod_{i=1}^{n}\left(\psi_{i}\right)^{k_{i}}\right)\left(\psi_{n+1}\right)^{k_{n+1}+1}\left(\psi_{n+2}\right)^{k_{n+2}+1}\right) \\
= & \left(\kappa_{k_{n+1}} \kappa_{k_{n+2}}+\kappa_{k_{n+1}+k_{n+2}}\right) \prod_{i=1}^{n}\left(\psi_{i}\right)^{k_{i}} .
\end{aligned}
$$

Proof. The proof follows from induction on $l$ and the following three geometric ingredients, which are well-known in the theory of moduli of curves:

- Let $\mathrm{ft}_{1}: \bar{M}_{g, n+1} \rightarrow \bar{M}_{g, n}$ be the forgetful morphism, forgetting the last marked point, and let $D_{i, n+1}$ be the boundary divisor in $\bar{M}_{g, n+1}$ defined as the image of the section of $\mathrm{ft}_{1}$, considered as the universal curve, by the $i$-th marked point. Then

$$
\mathrm{ft}_{1}^{*}\left(\psi_{i}\right)=\psi_{i}-D_{i, n+1} .
$$

- $\psi_{i}$ for $i \leq n$ on $\bar{M}_{g, n+1}$ vanishes when restricted to $D_{i, n+1}$.

$$
\mathrm{ft}_{1}^{*}\left(\kappa_{l}\right)=\kappa_{l}-\psi_{n+1}^{l} .
$$

The following result follows by combining the above ingredients and induction on power of $\kappa$-classes.

Corollary 1. A tautological equation on $\bar{M}_{g, n}$ involving $\kappa$-classes of highest power less than $l\left(\right.$ e.g. $\kappa_{k_{1}} \ldots \kappa_{k_{l}}$ ) can be written as a pushforward, via forgetful morphism $\mathrm{ft}^{l}$, of a tautological equation on $\bar{M}_{g, n+l}$, involving only boundary strata and $\psi$ classes.

By pulling back to moduli of stable maps, one has the following corollary. 
Corollary 2. (i) The system of generalized Gromov-Witten invariants involving $\kappa$ classes and $\lambda$-classes is the same as the system of usual Gromov-Witten invariants.

(ii) Any induced equation of generalized Gromov-Witten invariants can be written as an equation of ordinary Gromov-Witten invariants.

Proof. The fact that the system of generalized GW invariants involving $\lambda$-classes can be reduced to ordinary GW invariants follows from [7]. The part involving $\kappa$-classes follows from Lemma 2

With this corollary, one can talk about the induced equations of (ordinary) Gromov-Witten invariants from any tautological equations.

Remark 1. It is not difficult to see, from the above discussions (in particular equations (17), (18) and Lemma 21), that the three graphical operations introduced in [16] (cutting edges, genus reduction, and splitting vertices) are compatible with the pull-back operations.

\section{INVARIANCE UNDER LOWER TRIANGULAR SUBGROUPS}

The twisted loop group is generated by the "lower triangular subgroup" and the "upper triangular subgroup". The lower triangular subgroup consists of $\operatorname{End}(H)$ valued formal series $S\left(z^{-1}\right)=e^{s\left(z^{-1}\right)}$ in $z^{-1}$ satisfying $S^{*}(-z) S(z)=\mathbf{1}$ or equivalently

$$
s^{*}\left(-z^{-1}\right)+s\left(z^{-1}\right)=0 .
$$

5.1. Quantization of lower triangular subgroups. The quadratic hamiltonian of $s\left(z^{-1}\right)=\sum_{l=1}^{\infty} s_{l} z^{-l}$ is

$$
\sum_{l=1}^{\infty} \sum_{n=0}^{\infty} \sum_{i, j}\left(s_{l}\right)_{i j} q_{l+n}^{j} p_{n}^{i}+\sum \frac{1}{2}(-1)^{n}\left(s_{l}\right)_{i j} q_{n}^{i} q_{l-n-1}^{j} .
$$

The fact that $s\left(z^{-1}\right)$ is a series in $z^{-1}$ implies that the quadratic hamiltonian $P(s)$ of $s$ is of the form $q^{2}$-term $+q p$-term, where $q$ in the $q p$-term does not contain $q_{0}$. The quantization of the $P(s)$ is

$$
\hat{s}=\sum\left(s_{l}\right)_{i j} q_{l+n}^{j} \partial_{q_{n}^{i}}+\frac{1}{2 \hbar} \sum(-1)^{n}\left(s_{l}\right)_{i j} q_{n}^{i} q_{l-n-1}^{j} .
$$

Here $i, j$ are the indices of the orthonormal basis. (The indices $\mu, \nu$ will be reserved for the "gluing indices" at the nodes.) For simplicity of the notation, we adopt the summation convention to sum over all repeated indices.

Let $\frac{d \tau_{G}}{d \epsilon_{s}}:=\hat{s}(z) \tau_{G}$. Then

$$
\begin{aligned}
& \frac{d G_{0}\left(\epsilon_{s}\right)}{d \epsilon_{s}}=\sum_{l=1}^{\infty} \sum_{n=0}^{\infty} \sum_{i, j}\left(s_{l}\right)_{i j} q_{l+n}^{j} \partial_{q_{n}^{i}} G_{0}+\frac{1}{2}(-1)^{n}\left(s_{l+n+1}\right)_{i j} q_{n}^{i} q_{l}^{j}, \\
& \frac{d G_{g}\left(\epsilon_{s}\right)}{d \epsilon_{s}}=\sum_{l=1}^{\infty} \sum_{n=0}^{\infty} \sum_{i, j}\left(s_{l}\right)_{i j} q_{l+n}^{j} \partial_{q_{n}^{i}} G_{g}, \quad \text { for } g \geq 1 .
\end{aligned}
$$

Define

$$
\left\langle\partial_{k_{1}}^{i_{1}} \partial_{k_{2}}^{i_{2}} \ldots \partial_{k_{n}}^{i_{n}}\right\rangle_{g}:=\frac{\partial^{n} G_{g}}{\partial t_{k_{1}}^{i_{1}} \partial t_{k_{2}}^{i_{2}} \ldots \partial t_{k_{n}}^{i_{n}}},
$$


and denote $\langle\ldots\rangle:=\langle\ldots\rangle_{0}$. These functions $\langle\ldots\rangle_{g}$ will be called axiomatic GromovWitten invariants. Then

$$
\begin{aligned}
& \frac{d}{d \epsilon_{s}}\left\langle\partial_{k_{1}}^{i_{1}} \partial_{k_{2}}^{i_{2}} \ldots\right\rangle \\
= & \sum\left(s_{l}\right)_{i j} q_{l+n}^{j}\left\langle\partial_{n}^{i} \partial_{k_{1}}^{i_{1}} \ldots\right\rangle+\sum_{l=1}^{\infty} \sum_{i, a}\left(s_{l}\right)_{i i_{a}}\left\langle\partial_{k_{a}-l}^{i} \partial_{k_{1}}^{i_{1}} \ldots \partial_{k_{a}}^{\hat{i}_{a}} \ldots\right\rangle \\
+ & \frac{\delta}{2}\left((-1)^{k_{1}} \sum\left(s_{k_{1}+k_{2}+1}\right)_{i_{1} i_{2}}+(-1)^{k_{2}} \sum\left(s_{k_{1}+k_{2}+1}\right)_{i_{2} i_{1}}\right),
\end{aligned}
$$

where $\delta=0$ when there are more than 2 insertions and $\delta=1$ when there are two insertions. The notation $\hat{\partial}_{k}^{i}$ means that $\partial_{k}^{i}$ is omitted from the summation. We assume that there are at least two insertions, as this is the case in our application.

For $g \geq 1$

$$
\begin{aligned}
& \frac{d}{d \epsilon_{s}}\left\langle\partial_{k_{1}}^{i_{1}} \partial_{k_{2}}^{i_{2}} \ldots\right\rangle_{g} \\
= & \sum\left(s_{l}\right)_{i j} q_{l+n}^{j}\left\langle\partial_{n}^{i} \partial_{k_{1}}^{i_{1}} \ldots\right\rangle_{g}+\sum \sum_{a}\left(s_{l}\right)_{i i_{a}}\left\langle\partial_{k_{a}-l}^{i} \partial_{k_{1}}^{i_{1}} \ldots \partial_{k_{a}}^{\hat{i}_{a}} \ldots\right\rangle_{g} .
\end{aligned}
$$

\section{2. $S$-Invariance.}

Theorem 3 (S-invariance theorem). All tautological equations are invariant under action of lower triangular subgroups of the twisted loop groups.

Proof. Let $E=0$ be a tautological equation of axiomatic Gromov-Witten invariants. Suppose that this equation holds for a given semisimple Frobenius manifold, e.g. $H^{N p t} \cong \mathbb{C}^{N}$. We will show that $\hat{s} E=0$. This will prove the theorem.

$\hat{s} E=0$ follows from the following facts:

(a) The combined effect of the first term in (19) (for genus zero invariants) and in (20) (for $g \geq 1$ invariants) vanishes.

(b) The combined effect of the remaining terms in (19) and in (20) also vanishes.

(a) is due to the fact that the sum of the contributions from the first term is a derivative of the original equation $E=0$ with respect to $q$ variables. Therefore it vanishes.

It takes a little more work to show (b). Recall that all tautological equations are induced from moduli spaces of curves. Therefore, any relations of tautological classes on $\bar{M}_{g, n}$ contain no genus zero components of two or less marked points. However, when one writes the induced equation for (axiomatic) Gromov-Witten invariants, the genus zero invariants with two insertions will appear. This is due to the difference between the cotangent classes on $\bar{M}_{g, n+m}(X, \beta)$ and the pullback classes from $\bar{M}_{g, n}$. Therefore the only contribution from the third term of (19) comes from these terms. More precisely, let $\psi_{j}$ (descendents) denote the $j$ th cotangent class on $\bar{M}_{g, n+m}(X, \beta)$ and let $\bar{\psi}_{j}$ (ancestors) denote the pull-backs of cotangent classes from $\bar{M}_{g, n}$ by the combination of the stabilization and forgetful morphisms (forgetting the maps and extra marked points, and stabilizing if necessary).

Denote $\left\langle\partial_{k, \bar{l}}^{\mu}, \ldots\right\rangle$ as the generalized (axiomatic) Gromov-Witten invariants with $\psi_{1}^{k} \bar{\psi}_{1}^{l} \mathrm{ev}_{1}^{*}\left(\phi_{\mu}\right)$ at the first marked point. The equation (6) can be rephrased in terms 
of invariants as

$$
\left\langle\partial_{k, \bar{l}}^{i} \ldots\right\rangle_{g}=\left\langle\partial_{k+1, \overline{l-1}}^{i} \ldots\right\rangle_{g}-\left\langle\partial_{k}^{i} \partial^{\mu}\right\rangle g_{\mu \mu^{\prime}}\left\langle\partial_{\overline{l-1}}^{\mu^{\prime}} \ldots\right\rangle_{g} .
$$

For simplicity, denote

$$
\left\langle\ldots \partial^{\mu}\right\rangle\left\langle\partial^{\mu} \ldots\right\rangle:=\left\langle\ldots \partial^{\mu}\right\rangle g_{\mu \mu^{\prime}}\left\langle\partial^{\mu^{\prime}} \ldots\right\rangle .
$$

Repeating this process of reducing $\bar{l}$, one can show by induction that

$$
\begin{aligned}
& \left\langle\partial_{k, \bar{l}}^{i} \ldots\right\rangle_{g}=\left\langle\partial_{k+r, \overline{l-r}}^{i} \ldots\right\rangle_{g}-\left\langle\partial_{k+r-1}^{i} \partial^{\mu_{1}}\right\rangle\left\langle\partial_{\overline{l-r}}^{\mu_{1}} \ldots\right\rangle_{g}-\ldots \\
& -\left\langle\partial_{k}^{i} \partial^{\mu_{1}}\right\rangle\left[\sum_{p=1}^{r}(-1)^{p+1} \sum_{k_{1}+\ldots+k_{p}=r-p}\left\langle\partial_{k_{1}}^{\mu_{1}} \partial^{\mu_{2}}\right\rangle \ldots\left\langle\partial_{k_{p-1}}^{\mu_{p-1}} \partial^{\mu_{p}}\right\rangle\left\langle\partial_{k_{p}, \overline{l-r}}^{\mu_{p}} \ldots\right\rangle_{g}\right] .
\end{aligned}
$$

Now suppose that one has an equation of tautological classes of $\bar{M}_{g, n}$. Using the above equation (for $r=l$ ) one can translate the equation of tautological classes on $\bar{M}_{g, n}$ into an equation of the (axiomatic) Gromov-Witten invariants. The termwise cancellation of the contributions from the second and the third terms of (19) and (20) can easily be seen by straightforward computation.

If the above description is a bit abstract, the reader might want to try the following simple example: $\psi_{1}^{2}$ on $\bar{M}_{g, 1}$ is translated into invariants

$$
\left\langle\partial_{2}^{x}\right\rangle_{g}-\left\langle\partial_{1}^{x} \partial^{\mu}\right\rangle\left\langle\partial^{\mu}\right\rangle_{g}-\left\langle\partial^{x} \partial^{\mu}\right\rangle\left\langle\partial_{1}^{\mu}\right\rangle_{g}+\left\langle\partial^{x} \partial^{\mu}\right\rangle\left\langle\partial^{\mu} \partial^{\nu}\right\rangle\left\langle\partial^{\nu}\right\rangle_{g}
$$

The above "translation" from tautological classes to Gromov-Witten invariants are worked out explicitly in some examples in Sections 6 and 7 of [9].

Remark 2. The $S$-invariance theorem actually holds at the level of (Chow or cohomology) classes, rather than just the numerical invariants. The geometric content is (6). This should be clear from the proof.

5.3. Reduction to $q_{0}=0$. The arguments in this section are mostly taken from 13 .

Let $E=0$ be a tautological equation of (axiomatic) Gromov-Witten invariants. Since we have already proved $\hat{s}(E)=0$, our next goal would be to show $\hat{r}(E)=0$. In this section, we will show that it suffices to check $\hat{r}(E)=0$ on the subspace $q_{0}=0$.

Lemma 3. It suffices to show $\hat{r} E=0$ on each level set of the map $q \mapsto s$ in (10).

Proof. The union of the level sets is equal to $\mathcal{H}_{+}$.

Lemma 4. It suffices to check the relation for all $\hat{r}(z) E=0$ along $z \mathcal{H}_{+}$(i.e. $q_{0}=$ $0)$.

Proof. Theorem 5.1 of [1] states that a particular lower triangular matrix $S_{s}$, which is called a "calibration" of the Frobenius manifold, transforms the level set at $s$ to $z \mathcal{H}_{+}$. The $S$-invariance Theorem then concludes the proof.

Remark 3. In fact, $S_{s}$ can be taken as a fundamental solution of the horizontal sections of the Dubrovin (flat) connection, in $z^{-1}$ formal series. It was discovered in [11, following the works in [15] and [10, that

$$
\mathcal{A}:=\hat{S}_{s} \tau^{X}
$$

is the corresponding generating function for "ancestors". Therefore the transformed equation $\hat{S}_{s} E \hat{S}_{s}^{-1}=0$ is really an equation of ancestors. 


\section{INVARIANCE UNDER UPPER TRIANGULAR SUBGROUPS}

6.1. Quantization of upper triangular subgroups. The upper triangular subgroup consists of the regular part of the twisted loop groups $R(z)=e^{r(z)}$ satisfying

$$
R^{*}(-z) R(z)=\mathbf{1}
$$

or equivalently

$$
r^{*}(-z)+r(z)=0
$$

The quantization of $r(z)$ is

$$
\begin{aligned}
\hat{r}(z) & =\sum_{l=1}^{\infty} \sum_{n=0}^{\infty} \sum_{i, j}\left(r_{l}\right)_{i j} q_{n}^{j} \partial_{q_{n+l}^{i}} \\
& +\frac{\hbar}{2} \sum_{l=1}^{\infty} \sum_{m=0}^{l-1}(-1)^{m+1} \sum_{i j}\left(r_{l}\right)_{i j} \partial_{q_{l-1-m}^{i}} \partial_{q_{m}^{j}} .
\end{aligned}
$$

Therefore

$$
\begin{aligned}
& \frac{d\left\langle\partial_{k_{1}}^{i_{1}} \partial_{k_{2}}^{i_{2}} \ldots\right\rangle_{g}}{d \epsilon_{r}} \\
= & \sum_{l=1}^{\infty} \sum_{n=0}^{\infty} \sum_{i, j}\left(r_{l}\right)_{i j} q_{n}^{j}\left\langle\partial_{n+l}^{i} \partial_{k_{1}}^{i_{1}} \ldots\right\rangle_{g} \\
+ & \sum_{l=1}^{\infty} \sum_{i, a}\left(r_{l}\right)_{i i_{a}}\left\langle\partial_{k_{a}+l}^{i} \partial_{k_{1}}^{i_{1}} \ldots \partial_{k_{a}}^{i_{a}} \ldots\right\rangle_{g} \\
+ & \frac{1}{2} \sum_{l=1}^{\infty} \sum_{m=0}^{l-1}(-1)^{m+1} \sum_{i j}\left(r_{l}\right)_{i j}\left\langle\partial_{l-1-m}^{i} \partial_{m}^{j} \partial_{k_{1}}^{i_{1}} \partial_{k_{2}}^{i_{2}} \ldots\right\rangle_{g-1} \\
+ & \frac{1}{2} \sum_{l=1}^{\infty} \sum_{m=0}^{l-1}(-1)^{m+1} \sum_{i j} \sum_{g^{\prime}=0}^{g}\left(r_{l}\right)_{i j} \partial_{k_{1}}^{i_{1}} \partial_{k_{2}}^{i_{2}} \ldots\left(\left\langle\partial_{l-1-m}^{i}\right\rangle_{g^{\prime}}\left\langle\partial_{m}^{j}\right\rangle_{g-g^{\prime}}\right) .
\end{aligned}
$$

Here, if $g=0$, the third term on the right is $\langle\ldots\rangle_{-1}=0$ by definition. Also, it is understood that the formula for $\hat{r}_{l}$ extends to products of Gromov-Witten invariants by Leibniz's rule.

6.2. Relations to invariance of tautological equations. Let $E=0$ be a tautological equation on moduli of curves. As explained in Part I, it can be written in terms of a formal sum of decorated graphs. Denote $E=0$ also as the induced equation of Gromov-Witten invariants. Consider $\frac{d}{d \epsilon_{r}} E$. It is clear that the first term of (23) vanishes as $E=0$ implies $\sum\left(r_{l}\right)_{i j} q_{n}^{j} E=0$. Similarly, the contribution to the second term from $\hat{\partial}_{k}^{i}$ at an external marked point (i.e. not at a node) cancels. Therefore, $\frac{d}{d \epsilon_{r}} E$ consists of three parts: from the second term (with $\hat{\partial}_{k}^{i}$ at a node), third term and fourth term.

It follows from the usual correspondence between tautological classes and Gromov-Witten invariants that these three parts correspond to three graphical operations defined in [16]: 
- The second term, when $i_{a}$ are indices at a node, corresponds to cutting the edges.

- The third term corresponds to genus reduction.

- The last term corresponds to splitting the vertices.

Equation (22) implies that $\left(r_{l}\right)_{i j}$ is symmetric in $i, j$ for $l$ even and anti-symmetric for $l$ odd. The corresponding operation for a fixed $l$ on the decorated graphs is denoted $\mathfrak{r}_{l}$ in Part I. In fact, this is the original motivation of the Invariance Conjecture 1 .

To show that $\mathfrak{r}_{l}$ is well-defined is equivalent to showing

$$
\mathfrak{r}_{l}(E)=0
$$

for any tautological equation

$$
E=0
$$

in $R^{k}\left(\bar{M}_{g, n}\right)$. Using the link between $\mathfrak{r}_{l}$ on the moduli of curves and $\widehat{r}_{l}$ on GromovWitten theory, one's first step is to show that

$$
\widehat{r}_{l}(\tilde{E})=0
$$

for Gromov-Witten theory.

Note that equation (25) can be interpreted as an infinitesimal form of the requirement that the induced tautological equation

$$
\tilde{E}=0
$$

has the same form for any Gromov-Witten theory. That is, the tautological equation is invariant under the action of quantized upper triangular subgroups of the twisted loop groups. This is the reason the term "invariance of tautological equations" is used for equation (24).

\section{3. $R$-invariance.}

Theorem 4 (The $R$-invariance theorem). Equation (25) holds. That is, all tautological equations of Gromov-Witten invariants are invariant under the action of upper triangular subgroups of the twisted loop groups.

Proof. Since we have already established the $S$-invariance theorem, this theorem follows from Theorem 2. Indeed, (16) implies that there is a "loop group" element (or rather its quantization) taking a tautological equation on moduli of curves to that of any semisimple theory and vice versa. Therefore the set of induced tautological equations of one semisimple theory has a one-one correspondence with the set of tautological equations of another semisimple theory.

We remark again here that Theorem 4 holds at the level of Gromov-Witten classes in light of Teleman's result [20]. The infinitesimal form of Theorem 4 is the following theorem.

Theorem 5. The R-invariance theorem implies that (24) holds for any tautological equation. Equivalently, $\mathfrak{r}_{l}$ in (11) is well-defined at the level of tautological classes.

This in particular implies that Invariance Conjecture 1 in [16] is true.

Remark 4. As mentioned in the Introduction, Theorem 5 has a geometric interpretation which gives an imediate proof of this result. For a nice account of this interpretation, the readers are referred to [6], Section 3. The following proof is 
given here to show the interplay of the Gromov-Witten theory and the tautological rings of the moduli of curves.

Proof. With all the preparation above, there are two remaining ingredients in the proof:

(1) Teleman's classification theorem of semisimple cohomological field theories [20, applied to Givental's framework on $X=\mathbb{P}^{1}$.

(2) An explicit calculation of $r_{l}$-matrix of $\mathbb{P}^{1}$ in the Appendix.

Note that (1) is stronger than Theorem 2 it implies a "cycle form" of Givental's formula.

Let

$$
E=\sum_{i} c_{i} \Gamma_{i}=0
$$

be a tautological relation. (Notation as in Part I of [16.) The induced tautological equation on Gromov-Witten theory of two points is denoted $\tilde{E}$. (1) implies that this tautological equation on the (two copies of the) moduli space of curves $(X=2 p t)$ is transformed to the corresponding tautological equation on the moduli space of stable maps to $\mathbb{P}^{1}$. By Theorem 3, Remark 2 and (1) above,

$$
\frac{d}{d \epsilon_{r}} \tilde{E}=\sum_{l=1}^{\infty} \sum_{i, j=1}^{2}\left(r_{l}\right)_{i j} \mathfrak{r}_{l}^{i j} \tilde{E}=0,
$$

where $\mathfrak{r}_{l}^{i j}$ is the operation $\mathfrak{r}_{l}$, with the two new half-edges called $i$ and $j$ (for the $i$-th and $j$-th points). It remains to prove that $\left(r_{l}\right)_{i j}$ is "non-degenerate" in the sense that $\left(r_{l}\right)_{i j} \neq 0$ unless

$$
\mathfrak{r}_{l}^{i j} \tilde{E}=0
$$

due to the a priori constraint (22). The non-vanishing of the $r_{l}$-matrices follows from Proposition 2, which will be proved in the Appendix. Therefore,

$$
\mathfrak{r}_{l}^{i j} \tilde{E}=0,
$$

which implies (24).

6.4. Some applications of Theorem 5, In a series of joint work with D. Arcara [1, 2, 3] and with A. Givental [13, Theorem [5] is shown to imply that

- A uniform derivation of all known $g=1,2$ tautological equations.

- Derivation of a new tautological equation in $\bar{M}_{3,1}$ of codimension 3 .

- All monomials of $\kappa$-classes and $\psi$-classes are independent in $R^{k}\left(\bar{M}_{g, n}\right) /$ $R^{k}\left(\partial \bar{M}_{g, n}\right)$ for all $k \leq[g / 3]$.

The first two investigate the existence of tautological equations, while the last one deals with the non-existence of tautological equations. Theorem 5 provides a new and systematic way to study the existence or non-existence of tautological equations via induction and linear algebra. In fact, it is the simplicity of the derivations of the above results that might point to some new direction in the study of tautological rings. 


\section{Appendix A. The $R$ Matrix for $P^{1}$}

The notation here follows that in 19. All invariants and functions are for $X=\mathbb{P}^{1}$. Here

$$
R(z)=\sum_{n=0}^{\infty} R_{n} z^{n}
$$

is defined by $R_{0}=I$ (the $2 \times 2$ identity matrix) and the following recursive relation $(1.4 .5$ in 19 ):

$$
\begin{aligned}
& -\frac{\sqrt{-1}}{2}\left(u_{1}-u_{2}\right)^{-1}\left(\begin{array}{cc}
\left(R_{n-1}\right)_{1}^{2} & \left(R_{n-1}\right)_{2}^{2} \\
\left(R_{n-1}\right)_{1}^{1} & \left(R_{n-1}\right)_{2}^{1}
\end{array}\right)\left(d u_{1}-d u_{2}\right) \\
& \quad+\left(\begin{array}{ccc}
\left(d R_{n-1}\right)_{1}^{1} & \left(d R_{n-1}\right)_{2}^{1} \\
\left(d R_{n-1}\right)_{1}^{2} & \left(d R_{n-1}\right)_{2}^{2}
\end{array}\right)=\left(\begin{array}{cc}
0 & \left(R_{n}\right)_{2}^{1} \\
\left(R_{n}\right)_{1}^{2} & 0
\end{array}\right)\left(d u_{1}-d u_{2}\right)
\end{aligned}
$$

for $n \geq 1$, where $\left(u_{1}, u_{2}\right)$ are the canonical coordinates of $Q H^{*}\left(\mathbb{P}^{1}\right)$,

$$
u_{i}=\frac{1}{\sqrt{2}} t_{\mathbf{1}} \pm \frac{e^{\frac{t_{h}}{2}}}{\sqrt{2}} t_{\mathbf{h}} .
$$

\section{Proposition 1.}

$$
R_{n}=\left(\begin{array}{cc}
1 & 2 n(-1)^{n-1} \sqrt{-1} \\
2 n \sqrt{-1} & (-1)^{n}
\end{array}\right) \frac{c_{n}}{v^{n}},
$$

where

$$
c_{n}:=-\frac{\prod_{k=1}^{n-1}\left(-1+4 k^{2}\right)}{2^{2 n} n !}, \quad v:=u_{1}-u_{2},
$$

and it is understood that $c_{1}=-1 / 4$.

Proof. Equation (26) gives a recursive relation which determines all $R_{n}$ from $R_{0}=$ $I$. It is easy to check that (27) satisfies (26).

Recall that $r(z)$ is defined as $\log R(z)$.

Proposition 2.

$$
r_{l}=\left\{\begin{array}{cc}
\left(\begin{array}{cc}
0 & -c_{l} \sqrt{-1} \\
c_{l} \sqrt{-1} & 0
\end{array}\right) & \text { ifl is even, } \\
\left(\begin{array}{cc}
a_{l} & b_{l} \sqrt{-1} \\
b_{l} \sqrt{-1} & -a_{l}
\end{array}\right) & \text { ifl is odd, }
\end{array}\right.
$$

such that $a_{l}, b_{l}, c_{l}$ are all non-zero rational numbers.

The rest of the Appendix is used to prove this proposition.

First, it is not very difficult to see that the matrices $r_{l}$ should be of the above forms and that all $a_{l}, b_{l}, c_{l}$ are rational numbers. These assertions follow from the 
following formula for $r_{l}$ :

$$
\begin{aligned}
\sum_{l=1}^{\infty} r_{l} z^{l} & =\log R(z)=-\sum_{n=0}^{\infty} \frac{(1-R(z))^{n+1}}{n+1} \\
& =\sum_{l} z^{l} \sum_{m=1}^{l} \frac{(-1)^{m-1}}{m} \sum_{\substack{i_{1}+\cdots+i_{m}=l \\
i_{j}>0}} R_{i_{1}} \cdots R_{i_{m}},
\end{aligned}
$$

Equation (22), and induction on $l$.

Here are a few examples of $r_{l}$ matrices obtained from (28) and Proposition 1 ;

$$
\begin{aligned}
& r_{1}=\frac{1}{4}\left(\begin{array}{cc}
-1 & -2 \sqrt{-1} \\
-2 \sqrt{-1} & 1
\end{array}\right), \\
& r_{2}=\frac{1}{2^{3}}\left(\begin{array}{cc}
0 & 3 \sqrt{-1} \\
-3 \sqrt{-1} & 0
\end{array}\right) \\
& r_{3}=\frac{1}{2^{5}}\left(\begin{array}{cc}
-4 & -23 \sqrt{-1} \\
-23 \sqrt{-1} & 4
\end{array}\right), \\
& r_{4}=\frac{1}{2^{4}}\left(\begin{array}{cc}
0 & 33 \sqrt{-1} \\
-33 \sqrt{-1} & 0
\end{array}\right), \\
& r_{5}=\frac{1}{5 \cdot 2^{9}}\left(\begin{array}{cc}
-2132 & -20839 \sqrt{-1} \\
-20839 \sqrt{-1} & 2132
\end{array}\right) .
\end{aligned}
$$

The non-vanishing of $a_{l}, b_{l}, c_{l}$ requires some elementary (though somehow lengthy) arguments. The idea is to get some rough estimates of the absolute values of entries of $r_{l}$, which are enough to guarantee the non-vanishing. We will start with some preparations.

Define a function of $n, C(n)$, by

$$
C(n)=\prod_{k=1}^{n-1}\left(1-\frac{1}{4 k^{2}}\right) .
$$

Then for $n \geq 1$,

$$
\left(R_{n}\right)_{1}^{2}=C(n) \cdot(n-1) ! \cdot\left(\frac{-\sqrt{-1}}{2}\right) \cdot v^{-n} .
$$

Lemma 5. $C(n)$ is strictly decreasing and $\lim _{n \rightarrow \infty} C(n)>0.62$.

Proof. $C(n)$ is obviously strictly decreasing. Therefore

$$
\lim _{n \rightarrow \infty}[\ln C(n)]=\ln \left[\lim _{n \rightarrow \infty} C(n)\right] .
$$

Since

$$
\begin{gathered}
\ln (1-x) \geq 4\left(\ln \frac{3}{4}\right) x \quad \text { for } 0<x \leq \frac{1}{4}, \\
\lim _{n \rightarrow \infty}[\ln C(n)]=\lim _{n \rightarrow \infty}\left[\sum_{k=1}^{n-1} \ln \left(1-\frac{1}{4 k^{2}}\right)\right] \geq \frac{\pi^{2}}{6} \ln \frac{3}{4} .
\end{gathered}
$$

Therefore

$$
\lim _{n \rightarrow \infty} C(n) \geq e^{\frac{\pi^{2}}{6} \ln \frac{3}{4}} \approx 0.62299 \cdots
$$


By abuse of notation, let $\left|\left(R_{n}\right)_{i}^{j}\right|$ denote the absolute value of the coefficient of $v^{-n}$ in $\left(R_{n}\right)_{i}^{j}$. Since $\left(R_{n}\right)_{1}^{1}=\frac{-\sqrt{-1}}{2 n}\left(R_{n}\right)_{1}^{2}$, we get the following corollary:

Corollary 3. For $n \geq 1$,

$$
\begin{aligned}
& 0.62 \frac{(n-1) !}{2}<\left|\left(R_{n}\right)_{1}^{2}\right| \leq \frac{(n-1) !}{2}, \\
& 0.62 \frac{(n-1) !}{4 n}<\left|\left(R_{n}\right)_{1}^{1}\right| \leq \frac{(n-1) !}{4 n} .
\end{aligned}
$$

Now define $\sigma_{n}^{l}$ by

$$
\sigma_{n}^{l}:=\sum_{\substack{i_{1}+\cdots+i_{l}=n \\ i_{j}>0}} i_{1} ! i_{2} ! \cdots i_{l} !
$$

\section{Lemma 6.}

$$
\sigma_{n}^{l} \leq\left(\frac{8}{3}\right)^{l-1} n ! \quad \text { for } n=0,1,2,3, \cdots, \quad l=1,2,3, \cdots .
$$

Proof. It is obviously true for $l=1$ and arbitrary $n$. By induction on $n$ with fixed $l=2$,

$$
\sigma_{n}^{2} \leq \frac{8}{3} n !, \quad n=0,1,2,3, \cdots .
$$

Then we have induction on $l$. Assume that $\sigma_{n}^{l} \leq\left(\frac{8}{3}\right)^{l-1} n$ ! for all $n$. It suffices to show that $\sigma_{n}^{l+1} \leq\left(\frac{8}{3}\right)^{l} n$ ! for all $n$ :

$$
\begin{aligned}
\sigma_{n}^{l+1} & =0 ! \sigma_{n}^{l}+1 ! \sigma_{n-1}^{l}+2 ! \sigma_{n-2}^{l}+\cdots+(n-1) ! \sigma_{1}^{l}+n ! \sigma_{0}^{l} \\
& \leq\left(\frac{8}{3}\right)^{l-1}[0 ! n !+1 !(n-1) !+2 !(n-2) !+\cdots+(n-1) ! 1 !+n ! 0 !] \\
& =\left(\frac{8}{3}\right)^{l-1} \sigma_{n}^{2} \leq\left(\frac{8}{3}\right)^{l} n ! .
\end{aligned}
$$

Lemma 7. Let

$$
S(m):=\sum_{k=3}^{m} \frac{2^{k-3}(m-k) !}{k} \quad \text { for } m \geq 5
$$

Then

$$
T(m):=\frac{S(m)}{(m-3) !} \quad \text { is a decreasing function of } m \text { for } m \geq 5 .
$$

Proof. Clearly, the $i$-th term of $T(m) \geq i$-th term of $T(m+1)$ for $i=1,2, \cdots m-3$, with equality only for $i=1$. For $m \geq 5, T(m+1)<T(m)$ as

$$
\frac{2^{m-3}}{m(m-2) !}+\frac{2^{m-2}}{(m+1)(m-2) !}<\frac{2^{m-3}}{m(m-3) !} .
$$

Corollary 4. (1) $T(m)<1 \quad$ for $m \geq 5$.

(2) $T(m)<0.477$ for $m \geq 9$.

(3) $S(m)<(m-3)$ ! for $m \geq 5$.

(4) $S(m)<0.477(m-3)$ ! for $m \geq 9$. 


\section{Corollary 5.}

$$
\frac{1}{2}\left|\sum_{k=1}^{m-1}\left(R_{k} R_{m-k}\right)_{1}^{1}\right| \leq \frac{15}{256}(m-3) \text { ! for } m \text { odd, } m \geq 7 .
$$

Proof. Since $m$ is odd,

$$
\begin{aligned}
& \left.\frac{1}{2}\left|\sum_{k=1}^{m-1}\left(R_{k} R_{m-k}\right)_{1}^{1}\right| \leq \sum_{k=1}^{\frac{m-1}{2}}\left|\left(R_{k}\right)_{1}^{1}\right| \mid R_{m-k}\right)_{1}^{1} \mid \\
\leq & \frac{1}{4} \cdot \frac{3(m-2) !}{16(m-1)}+\frac{3 \cdot 1 !}{16 \cdot 2} \cdot \frac{3(m-3) !}{16(m-2)}+\cdots+\frac{3\left(\frac{m-3}{2}\right) !}{16\left(\frac{m-1}{2}\right)} \cdot \frac{3\left(\frac{m-1}{2}\right) !}{16\left(\frac{m+1)}{2}\right)} \\
\leq & \frac{3}{64}(m-3) !+\frac{9}{256}\left[0 !(m-4) !+1 !(m-5) !+\cdots+\left(\frac{m-5}{2}\right) !\left(\frac{m-3}{2}\right) !\right] \\
\leq & \frac{3}{64} \cdot \frac{5}{4} \cdot(m-3) !=\frac{15}{256}(m-3) ! \quad \text { for } m \geq 7 .
\end{aligned}
$$

\section{Lemma 8.}

$$
\left|\left(R_{m}\right)_{1}^{1}\right| \geq 0.13(m-2) ! \quad \text { for } m \geq 7 .
$$

Proof. By Corollary 3

$$
\begin{aligned}
\left|\left(R_{m}\right)_{1}^{1}\right| & >0.62 \cdot \frac{(m-1) !}{4 m}=\frac{1}{4} \cdot 0.62 \cdot\left(1-\frac{1}{m}\right)(m-2) ! \\
& \geq \frac{1}{4} \cdot 0.62 \cdot \frac{6}{7}(m-2) ! \\
& >0.13(m-2) !
\end{aligned}
$$

Now we are ready to prove Proposition 2, We start with off-diagonal entries:

$$
\left(r_{l}\right)_{1}^{2}=\left(R_{l}\right)_{1}^{2}+\underbrace{\sum_{n=2}^{l} \frac{(-1)^{n-1}}{n} \sum_{\substack{i_{1}+\cdots+i_{n}=l \\ i_{j}>0}}\left(R_{i_{1}} \cdots R_{i_{n}}\right)_{1}^{2}}_{=:\left(R_{l}^{\prime}\right)_{1}^{2}} .
$$

By the triangular inequality, we have

$$
\left|\left(r_{l}\right)_{1}^{2}\right| \geq||\left(R_{l}\right)_{1}^{2}|-|\left(R_{l}^{\prime}\right)_{1}^{2}||
$$

so it suffices to show

$$
\left|\left(R_{l}\right)_{1}^{2}\right|>\left|\left(R_{l}^{\prime}\right)_{1}^{2}\right|
$$


Now,

$$
\begin{aligned}
& \left|\left(R_{l}^{\prime}\right)_{1}^{2}\right| \\
\leq & \sum_{n=2}^{l} \frac{1}{n} \sum_{\substack{i_{1}+\cdots+i_{n}=l \\
i_{j}>0}}\left|\left(R_{i_{1}} \cdots R_{i_{n}}\right)_{1}^{2}\right| \\
\leq & \sum_{n=2}^{l} \frac{1}{n} \sum \frac{\left(i_{j_{1}}-1\right) !}{2} \cdots \frac{\left(i_{j_{k}}-1\right) !}{2} \cdot \frac{\left(i_{j_{k+1}}-1\right) !}{4 i_{j_{k+1}}} \cdots \frac{\left(i_{j_{n}}-1\right) !}{4 i_{j_{n}}} \quad \text { by Corollary } 3 \text { ) } \\
\leq & \sum_{n=2}^{l} \frac{1}{n} \cdot \frac{3^{n}-(-1)^{n}}{2 \cdot 4^{n}} \cdot\left(\frac{8}{3}\right)^{n-1}(l-n) ! \quad \text { (by Lemma }[6) \\
= & \frac{3}{16} \sum_{n=2}^{l} \frac{1}{n}\left[2^{n}-\left(-\frac{2}{3}\right)^{n}\right](l-n) ! \\
< & \frac{3}{16} \sum_{n=2}^{l} \frac{1}{n} \cdot 2^{n} \cdot(l-n) ! \\
< & \frac{3}{8}(l-2) !+\frac{3}{2}(l-3) ! \quad \text { for } l \geq 1 \quad \text { (by Corollary 41). }
\end{aligned}
$$

By Corollary 3 ,

$$
0.31(l-1) !<\left|\left(R_{l}\right)_{1}^{2}\right|<0.5(l-1) \text { ! for } l \geq 1 .
$$

So for $l \geq 5$,

$$
\left|\left(R_{l}\right)_{1}^{2}\right|>0.3(l-1) !>\frac{3}{8}(l-2) !+\frac{3}{2}(l-3) !>\left|\left(R_{l}^{\prime}\right)_{1}^{2}\right|,
$$

i.e., $\left(r_{l}\right)_{1}^{2} \neq 0$. Since we know $\left(r_{l}\right)_{1}^{2} \neq 0$ for $l=1,2,3,4,\left(r_{l}\right)_{1}^{2} \neq 0$ for all $l \geq 1$.

Similarly for diagonal terms for $l=o d d$ :

$$
\begin{aligned}
\left|\left(R_{l}^{\prime}\right)_{1}^{1}\right| & \leq \frac{1}{2} \sum_{\substack{i_{1}+i_{2}=l \\
i_{j}>0}}\left|\left(R_{i_{1}} R_{i_{2}}\right)_{1}^{1}\right|+\sum_{n=3}^{l} \frac{1}{n} \sum_{\substack{i_{1}+\ldots+i_{n}=l \\
i_{j}>0}}\left|\left(R_{i_{1}} \cdots R_{i_{n}}\right)_{1}^{1}\right| \\
& \leq \frac{15}{256}(l-3) !+\frac{3}{16} \sum_{n=3}^{l} \frac{1}{n}\left[2^{n}+\left(-\frac{2}{3}\right)^{n}\right](l-n) ! \\
& <\frac{15}{256}(l-3) !+\frac{3}{16} \sum_{n=3}^{l} \frac{1}{n} \cdot 2^{n} \cdot(l-n) ! \\
& =\frac{15}{256}(l-3) !+\frac{3}{2} S(l) \\
& \left.<\frac{15}{256}(l-3) !+0.72(l-3) ! \quad \text { for } l \geq 9, m=\text { odd } \quad \text { (by Corollary } 4\right) .
\end{aligned}
$$

By Lemma 8 .

$$
\left|\left(R_{l}\right)_{1}^{1}\right|>0.13(l-1) !>\left(\frac{15}{256}+0.72\right)(l-3) !>\left|\left(R_{l}^{\prime}\right)_{1}^{1}\right| \quad \text { for } l \geq 9 .
$$

Thus $\left(r_{l}\right)_{1}^{1} \neq 0$ for $l \geq 9, l=$ odd. We know $\left(r_{l}\right)_{1}^{1} \neq 0$ for $l=1,3,5$. 
It is now left to check $l=7$, which is checked by hand:

$$
\begin{aligned}
\left|\left(R_{7}^{\prime}\right)_{1}^{1}\right| & \leq \frac{15}{256} \cdot 4 !+\sum_{m=3}^{7} \frac{3^{m}+(-1)^{m}}{2 \cdot 4^{m}} \cdot \sigma_{7-m}^{m} \\
& =\frac{15 \cdot 24}{256}+\frac{1}{2}\left[\frac{1}{3} \cdot \frac{27-1}{64} \cdot 108+\frac{1}{4} \cdot \frac{81+1}{256} \cdot 52\right. \\
& \left.+\frac{1}{5} \cdot \frac{243-1}{1024} \cdot 20+\frac{1}{6} \cdot \frac{729+1}{4096} \cdot 6+\frac{1}{7} \cdot \frac{2187-1}{16384} \cdot 1\right]=11.3720 \cdots
\end{aligned}
$$

On the other hand,

$$
\left|\left(R_{7}\right)_{1}^{1}\right|=\frac{3 \cdot 15 \cdot 35 \cdot 63 \cdot 99 \cdot 143}{2^{14} \cdot 7 !}=17.0114 \cdots .
$$

So $\left(r_{7}\right)_{1}^{1} \neq 0$. The proof of Proposition 2 is now complete.

\section{ACKNOWLEDGMENTS}

I wish to thank D. Arcara, E. Getzler, A. Givental, R. Pandharipande, C. Teleman and R. Vakil for many useful discussions.

\section{REFERENCES}

[1] D. Arcara, Y.-P. Lee, Tautological equations in genus two via invariance constraints, Bull. Inst. Math. Acad. Sin. (N.S.) 2 (2007), no. 1, 1-27. MR2294106 (2008a:14068)

[2] D. Arcara, Y.-P. Lee, Tautological equation in $\bar{M}_{3,1}$ via invariance constraints, math.AG/0503184, to appear in Canadian Mathematical Bulletin.

[3] D. Arcara, Y.-P. Lee, On independence of generators of the tautological rings, math.AG/0605488, to appear in Compositio Math.

[4] T. Coates, A. Givental, Quantum Riemann - Roch, Lefschetz and Serre, Ann. of Math. (2) 165 (2007), no. 1, 15-53. MR2276766 (2007k:14113)

[5] R. Dijkgraaf, E. Witten, Mean field theory, topological field theory, and multi-matrix models, Nuclear Phys. B 342 (1990), no. 3, 486-522. MR1072731 (92i:81271)

[6] C. Faber, S. Shadrin, D. Zvonkine, Tautological relations and the r-spin Witten conjecture, arXiv:math/0612510.

[7] C. Faber, R. Pandharipande, Hodge integrals and Gromov-Witten theory, Invent. Math. 139 (2000), no. 1, 173-199. MR.1728879 (2000m:14057)

[8] C. Faber, R. Pandharipande, Relative maps and tautological classes, J. Eur. Math. Soc. (JEMS) 7 (2005), no. 1, 13-49. MR2120989 (2005m:14046)

[9] E. Getzler, Topological recursion relations in genus 2, Integrable systems and algebraic geometry (Kobe/Kyoto, 1997), 73-106, World Sci. Publishing, River Edge, NJ, 1998. MR1672112 (2000b:14028)

[10] E. Getzler, The jet-space of a Frobenius manifold and higher-genus Gromov-Witten invariants, Frobenius manifolds, 45-89, Aspects Math., E36, Vieweg, Wiesbaden, 2004. MR2115766 (2006i:53122)

[11] A. Givental, Gromov-Witten invariants and quantization of quadratic Hamiltonians, Dedicated to the memory of I. G. Petrovskii on the occasion of his 100th anniversary. Mosc. Math. J. 1 (2001), no. 4, 551-568, 645. MR1901075 (2003j:53138)

[12] A. Givental, Symplectic geometry of Frobenius structures, Frobenius manifolds, 91-112, Aspects Math., E36, Vieweg, Wiesbaden, 2004. MR2115767 (2005m:53172)

[13] A. Givental, Y.-P. Lee, unpublished.

[14] M. Kontsevich, Yu. Manin, Gromov-Witten classes, quantum cohomology, and enumerative geometry, Comm. Math. Phys. 164 (1994), no. 3, 525-562. MR.1291244(95i:14049)

[15] M. Kontsevich, Yu. Manin, Relations between the correlators of the topological sigma-model coupled to gravity, Comm. Math. Phys. 196 (1998), no. 2, 385-398. MR 1645019 (99k:14040)

[16] Y.-P. Lee, Invariance of tautological equations I: Conjectures and applications, J. Eur. Math. Soc. (JEMS) 10 (2008), no. 2, 399-413. MR2390329 
[17] Y.-P. Lee, Witten's conjecture, Virasoro conjecture, and invariance of tautological equations, math.AG/0311100.

[18] Y.-P. Lee, Witten's conjecture and Virasoro conjecture up to genus two, Gromov-Witten theory of spin curves and orbifolds, 31-42, Contemp. Math., 403, Amer. Math. Soc., Providence, RI, 2006. MR2234883(2007e:14089)

[19] Y.-P. Lee, R. Pandharipande, Frobenius manifolds, Gromov-Witten theory, and Virasoro constraints, in preparation. Materials needed for this paper are available from http://www.math.princeton.edu/ rahulp/

[20] C. Teleman, The structure of 2D semi-simple field theories, arXiv:0712.0160.

[21] R. Vakil, The moduli space of curves and Gromov-Witten theory, math.AG/0602347.

Department of Mathematics, University of Utah, Salt Lake City, Utah 84112-0090

E-mail address: yplee@math.utah.edu

Department of Mathematics, University of Utah, Salt Lake City, Utah 84112-0090

E-mail address: yshr19@gmail.com 